\title{
Investigation of surface properties of Si-doped GaN films by electric force microscopy and photoluminescence
}

\author{
T.Y. Lin ${ }^{\mathrm{a}, *}$, W.S. Su ${ }^{\mathrm{a}}$, W.S. Su ${ }^{\mathrm{b}}$, Y.F. Chen ${ }^{\mathrm{b}}$ \\ anstitute of Optoelectronic Sciences, National Taiwan Ocean University, Keelung, Taiwan, ROC \\ ${ }^{\mathrm{b}}$ Department of Physics, National Taiwan University, Taipei, Taiwan, ROC
}

Received 29 December 2003; accepted 10 January 2004 by P. Wachter

\begin{abstract}
Results of the electric force microscopy (EFM) and photoluminescence (PL) measurements in a set of Si-doped GaN films are presented. The EFM measurements indicate that substantial surface states exist in GaN thin films and the density of surface states is reduced with the Si doping concentration. It is found that this result strongly correlates with the optical properties of the epifilms. We show that the ratio of near-band edge luminescence of the front-side and back-side PL spectra increases with the doping concentration. This behavior manifests the effects of the surface states on the luminescent properties of GaN. Our investigation therefore not only shows the important role of surface states on the optical properties of GaN, but it also reveals that the combination of EFM and optical techniques is a very powerful way to understand the physical properties of a thin film. (C) 2004 Elsevier Ltd. All rights reserved.
\end{abstract}

PACS: $78.66-\mathrm{w} ; 78.55-\mathrm{m} ; 73.20-\mathrm{r}$

Keywords: A. III-V semiconductors; D. Optical properties; D. Surface states

$\mathrm{GaN}$ and related heterostructures are now well established as materials of importance to both science and technology for the development of high-temperature microelectronics, light emitters, and detectors [1,2]. Great attention was paid to the emission mechanism in the active layer of the emitting devices, both experimentally $[3,4]$ and theoretically $[5,6]$. On the other hand, fewer investigations have focused on surface properties of $\mathrm{GaN}$ [7-11], which are very important to the reliable performance for the device applications. The $\mathrm{n}-/ \mathrm{p}$ - type nature of $\mathrm{GaN}$ and $\mathrm{InGaN}$ layers, and their related band bendings in surface and interfaces have been studied by contactless electroreflectance [7]. It was found that the effect of surface layer is closely related to the optical properties of $\mathrm{GaN}$ upon annealing [8] or after surface treatment $[9,10]$. Besides, the existence of the surface state has been considered as the possible reason for the observation of persistent photoluminescence (PL) in $\mathrm{GaN}$

\footnotetext{
* Corresponding author. Tel.: +886-2246-22192-6703; fax: +886-224-634-360.

E-mail address: tylin@mail.ntou.edu.tw (T.Y. Lin).
}

[11]. Recently, there have been several reports concerning the electric force microscopy (EFM) on GaN samples [12-16]. The surface potential mode of EFM is very sensitive to the polarity of the surface charge and has been used to image the inversion domains in GaN [12]. The piezoelectrically induced surface charge and potential can also be measured by EFM [13]. Accordingly, it is interesting to combine the EFM and optical techniques to study the surface properties of $\mathrm{GaN}$, which are important to optical and electrical performance of GaN-based devices.

In this article, we present EFM measurements and the results of the front-side and back-side PL measurements in a set of the Si-doped GaN epifilms. We demonstrate that EFM technique is able to detect the effect of Si doping on the surface properties of GaN films. Our experimental results reveal that substantial surface states exist in $\mathrm{GaN}$ epifilms, and they can be passivated by the Si incorporation. Our results also show that the defects near the surface are responsible for the inefficient band-edge emission observed in the PL spectra. In view of effects of surface related defects on the electrical and optical properties of III-V 
nitride materials, the results shown here should be very important for their related devices.

The Si-doped GaN films used here were grown by metalorganic chemical vapor deposition on $c$-face sapphire substrates. A thin GaN buffer layer $(\sim 500 \AA)$ was deposited at $500{ }^{\circ} \mathrm{C}$ right before the epitaxial $\mathrm{GaN}(\sim 2 \mu \mathrm{m})$ grown at $1000^{\circ} \mathrm{C}$. The samples in a set had exactly the same growth conditions except for the silane $\left(\mathrm{SiH}_{4}\right)$ flow rate to achieve doping densities in the range from $4.5 \times 10^{17}$ to $5.2 \times 10^{18} \mathrm{~cm}^{-3}$. All samples used here were cleaned initially with trichloroethylene, acetone, and methanol in ultrasonic bath. For the PL measurements, the samples were excited from either the $\mathrm{GaN}$ side (front-side) or the sapphire side (back-side) by a $50 \mathrm{~mW} / \mathrm{cm}^{2} \mathrm{He}-\mathrm{Cd}$ laser emitting at $325 \mathrm{~nm}$. A $0.85 \mathrm{~m}$ monochromator, a photomultiplier tube, and standard lock-in technique were used to detect the signals.

EFM measurements were carried out using a Stand Alone SMENA apparatus (NT-MDT) in a tapping mode under ambient conditions. The silicon tips of tapping mode were coated by a variety of different metals to vary the metal work function for EFM. The EFM was performed by measuring the electrostatic forces from the samples with a voltage applied to the metal-coated silicon tips. Variations in the electrostatic force are sensed by the electric field gradient, which are monitored by resonant frequency change in the oscillating tips. The tips are scanned across the surface at a constant tip-sample separation $(30 \mathrm{~nm})$ in this study.

The total electrostatic force acting on the tip $F$ is due to the charge-charge interaction, and change in the capacitance energy stored in the tip-sample capacitance [17]. When a dc bias is applied to the metal tip, the total force on the tip is therefore given by $[13,14,17]$

$F=\frac{q_{\mathrm{s}} q_{\mathrm{t}}}{4 \pi \varepsilon_{0} z^{2}}+\frac{1}{2} \frac{\mathrm{d} C}{\mathrm{~d} z}\left(V_{\text {applied }}-V_{\text {contact }}\right)^{2}$

where $C$ is the capacitance between the tip and sample, $z$ is the tip-sample separation, $q_{\mathrm{s}}$ is the surface charge, $q_{\mathrm{t}}$ is the charge induced on the tip, $q V_{\text {contact }}$ is the difference between the Fermi levels of the sample and the tip metallization, and note that $V_{\text {contact }}=\phi_{\mathrm{m}}-\chi_{\mathrm{GaN}}-\phi_{\mathrm{Bn}}-\Delta \phi$. In this formula, $\phi_{\mathrm{m}}$ is the metal work function, $\chi_{\mathrm{GaN}}$ is the electron affinity of GaN [18], $\Delta \phi$ is the band bending caused by surface states, and $\phi_{\mathrm{Bn}}$ is the barrier height of metalsemiconductor barrier.

The EFM images of the surface of the Si-doped $\mathrm{GaN}$ films are shown in Fig. 1. The electrostatic force is characterized by the contrast of EFM signal or the rootmean-square (rms) roughness of the signal. When the tip bias is increased gradually, the contrast of EFM signal will change from high to the lowest. The lowest EFM signal signifies a force minimum when the applied bias is equal to the contact potential between the $\mathrm{GaN}$ and the tip metallization as indicated by Eq. (1). We can get a tip voltage called null voltage $V_{\text {null }}$ responsible for the minimum force condition. It is found that the electrostatic force varied with the magnitude of the tip voltage and not the sign, consistent with the theoretical $V^{2}$ dependence [13, 17]. From Fig. 1, we can observe experimentally a $V_{\text {null }}$ of $0.5 \mathrm{~V}$ for a n-GaN with carrier concentration of $1.06 \times 10^{18} \mathrm{~cm}^{-3}$ in the case of Pt coated tips. The inset of Fig. 2 is a plot of the signal rms roughness as a measure of contrast against the tip voltage to illustrate the minimum force condition.

The measured $V_{\text {null }}$ or the contact potential between the tip metallization and $\mathrm{GaN}$ material is a function of the difference between metal work function and electron affinity of $\mathrm{GaN}$ according to the Cowley and Sze model [19],

$$
\begin{gathered}
\phi_{\mathrm{Bn}}=a\left(\phi_{\mathrm{m}}-\chi_{\mathrm{GaN}}\right)+(1-a)\left(\frac{E_{\mathrm{g}}}{q}-\phi_{0}\right)-\Delta \phi \\
=a\left(\phi_{\mathrm{m}}-\chi_{\mathrm{GaN}}\right)+b \\
V_{\text {contact }}=(1-a)\left(\phi_{\mathrm{m}}-\chi_{\mathrm{GaN}}\right)-(b+\Delta \phi)
\end{gathered}
$$

where $\phi_{\mathrm{Bn}}$ is the barrier height of metal-semiconductor, $\phi_{\mathrm{m}}$ is the work function of metal, $\chi_{\mathrm{GaN}}$ is the electron affinity of semiconductor, $E_{\mathrm{g}}$ is the band gap, $\Delta \phi$ is the image force barrier lowing, $\phi_{0}$ is the energy level at surface relative to the valence band, $a$ and $b$ are quantities related to the slope and intercept in Eq. (3). Fig. 2 is a plot of $\phi_{\mathrm{m}}-\chi_{\mathrm{GaN}}$ dependence on the measured tip null voltage $V_{\text {null }}$. With a least squares linear fit of the experimental results, we found a slope of 0.19 and an intercept of -0.10 . The density of surface states $D_{\mathrm{s}}$ can now be calculated with the values of the slope and the intercept, as well as the dielectric constant $\varepsilon_{i}$, and the thickness $\delta$, of the metal-semiconductor interface in the following expressions,

$D_{\mathrm{s}}=\frac{(1-a) \varepsilon_{i}}{a \delta q^{2}}$

$\phi_{0}=\frac{E_{\mathrm{g}}}{q}-\frac{b+\Delta \phi}{1-a}$

It was found that the density of surface states decreases and the surface state energy increases as the doping concentration increases. Using the air gap as the dielectric for the interface, the calculated density of surface states is $4.30 \times 10^{10} \mathrm{~cm}^{-2} \mathrm{eV}^{-1}$ with $\phi_{0}=2.86 \mathrm{eV}$ for the sample with the highest doping concentration $\left(N_{\mathrm{D}}-N_{\mathrm{A}}=5.2 \times\right.$ $\left.10^{18} \mathrm{~cm}^{-3}\right)$. For the sample with the lowest doping concentration $\left(N_{\mathrm{D}}-N_{\mathrm{A}}=4.5 \times 10^{17} \mathrm{~cm}^{-3}\right)$, the surface state density was found to be $1.87 \times 10^{11} \mathrm{~cm}^{-2} \mathrm{eV}^{-1}$ with $\phi_{0}=2.58 \mathrm{eV}$. Our result reveals that substantial surface states in $\mathrm{GaN}$ epifilms can be passivated by the Si dopants. Note that the surface state energy $\phi_{0}$ specifies the energy level below which all surface states must be filled for the charge neutrality at the surface. Thus, one of the reasons that the surface state energy increases with doping concentration is due to the decrease in the density of surface states.

In order to probe the influence of surface states on the optical properties of GaN films, we have performed the PL measurements. Inset of Fig. 3 shows the front-side and back- 

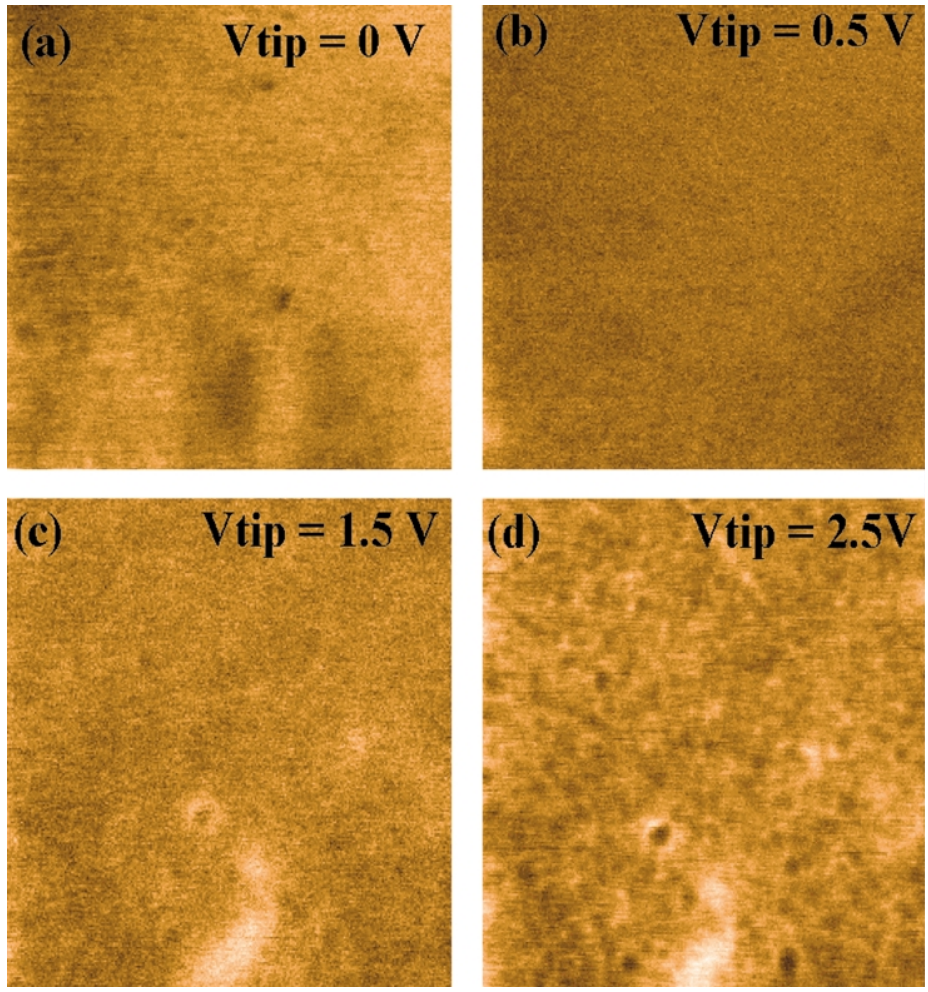

Fig. 1. Electrostatic force $(2 \mu \mathrm{m} \times 2 \mu \mathrm{m})$ image of the surface of a Si-doped GaN with carrier concentration of $1.06 \times 10^{18} \mathrm{~cm}^{-3}$ as a function of tip applied voltage. Scan (a)-(d) are the EFM data with the tip bias increasing from (a) to (d). Note that the $V_{\text {null }}=0.5 \mathrm{~V}$ in the case of platinum coated tips.

side PL spectra taken from one of the Si-doped GaN epifilms at room temperature. Both the front-side and back-side spectra show the near-band edge ultraviolet (UV) transition at $3.418 \mathrm{eV}$ and the broad yellow luminescence (YL) centered around $2.2 \mathrm{eV}$. Previously, we have shown that the defects responsible for the back-side PL spectra exist mainly near the interface between the buffer layer and the

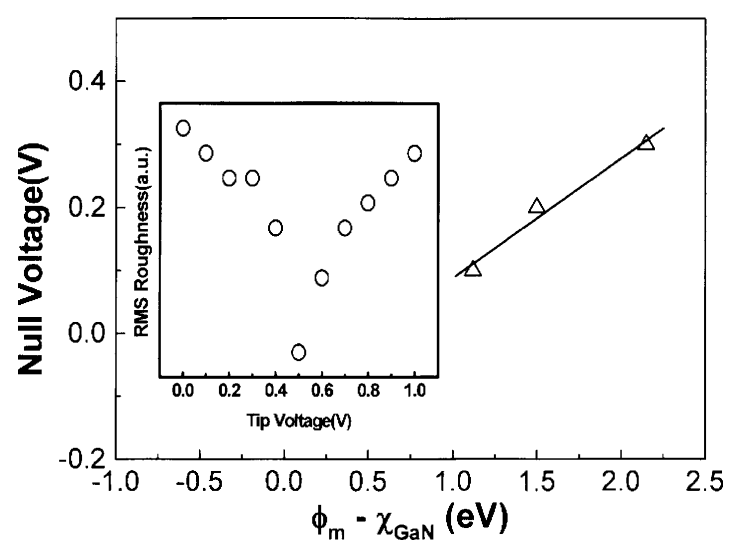

Fig. 2. Plot of the tip voltage for a minimum force condition vs the difference between metal work function and electron affinity of GaN. Inset: Plot of the rms contrast against the tip bias illustrating the force minimum condition for the platinum coated tip. epilayer [20]. In addition, the density of the acceptor like deep level near the interface region does not depend on $\mathrm{Si}$ doping concentration. On the other hand, the near-band edge transition of the front side is likely to be much more sensitive to changes of the surface states because the photo-

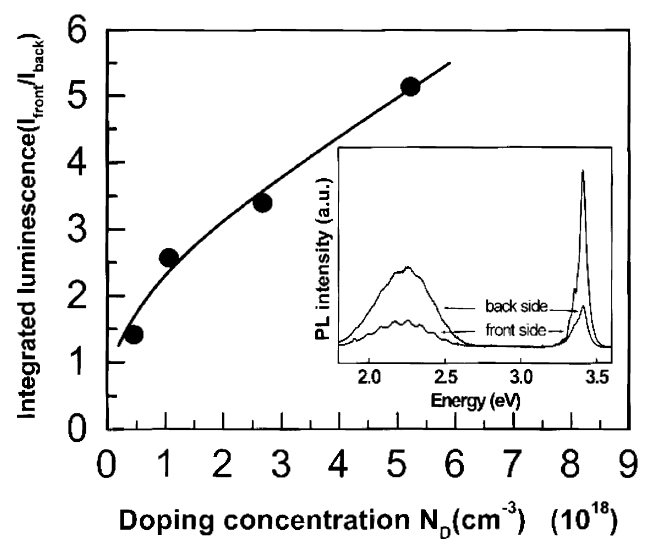

Fig. 3. The UV PL spectra ratio of the front-side to the back-side as a function of doping concentration. The room-temperature spectra of Si-doped GaN epitaxial film are shown in the inset. The front-side PL spectrum is excited from the GaN side and the back-side PL spectrum is excited from the sapphire side. Only one of the Si-doped $\mathrm{GaN}$ sample is shown here. 
excited electron-hoe pairs are within $100 \mathrm{~nm}$ away from the surface. Therefore, the changes in optical properties due to surface states can be illustrated by the relative weights of the front-side and back-side luminescence components. Fig. 3 shows the UV PL spectra ratio of the front-side to the backside as a function of Si doping concentration. It is found that the integrated luminescence ratio increases as the doping concentration increases. Considering that the front-side PL intensity is very sensitive to surface recombination velocity, the increased PL ratio of the UV luminescence is indicative of a reduction in surface recombination velocity due to the changes of surface states. Indeed, the Si doping has been found to be able to suppress the formation of point defects in $\mathrm{GaN}$ and prevent from the potential irregularities at the domain boundaries in Si doped layers [21]. Thus, we point out that the defects near the surface play an important role in emission mechanisms in GaN thin films [11] and are responsible for the inefficient band-edge emission observed in the PL spectra.

In conclusion, we have reported the surface property of Si-doped GaN films by EFM and its influence on PL spectra. The EFM results indicate that substantial surface states exist in GaN thin films and their density decreases with Si doping concentration. The effects of the surface states on the luminescent properties of $\mathrm{GaN}$ epifilms are manifested by the front-side and back-side PL spectra taken from a series of samples. It is found that the surface defects do play an important role in determining the efficiency of the bandedge emission. In view of the effect of surface defects on the electrical and optical properties of III-V nitride materials, the results shown here should be very important for the application of GaN-based devices.

\section{Acknowledgements}

This research was partially supported by the National Science Council of ROC.

\section{References}

[1] H. Morkoc (Eds.), Nitride Semiconductors and Devices, Springer, Berlin, 1999.

[2] B. Gil (Eds.), Group III Nitride Semiconductor Compounds: Physics and Applications, Clarendon Press, Oxford, 1998.

[3] Y. Narukawa, Y. Kawakami, S. Fujita, S. Nakamura, Phys. Rev. 59 (1999) 10283.

[4] P. Waltereit, O. Brandt, J. Ringling, H. Ploog, Phys. Rev. 64 (2001) 245305.

[5] I. Ho, G.B. Stringfellow, Appl. Phys. Lett. 69 (1996) 2701.

[6] F. Bernardini, V. Fiorentini, D. Vanderbilt, Phys. Rev. B 63 (2001) 193201.

[7] W. Krystek, F.H. Pollak, Z.C. Feng, M. Schurman, R.A. Stall, Appl. Phys. Lett. 72 (1998) 1353.

[8] E. Oh, B. Kim, H. Park, Y. Park, Appl. Phys. Lett. 73 (1998) 1883.

[9] M.A. Reshchikov, P. Visconti, K.M. Jones, H. Morkoc, Mater. Res. Soc. Symp. Proc. 680E (2001) E5.4.

[10] J.O. Song, S.J. Park, T.Y. Seong, Appl. Phys. Lett. 80 (2002) 3129.

[11] M.A. Reshchikov, M. Zafar Iqbal, H. Morkoc, S.S. Park, K.Y. Lee, Appl. Phys. Lett. 83 (2003) 266.

[12] K.M. Jones, P. Visconti, F. Yun, A.A. Baski, H. Morkoc, Appl. Phys. Lett. 78 (2001) 2497.

[13] P.M. Bridger, Z.Z. Bandic, E.C. Piquette, T.C. McGill, Appl. Phys. Lett. 74 (1999) 3522.

[14] G. Koley, M.G. Spencer, J. Appl. Phys. 90 (2001) 337.

[15] J.W.P. Hsu, H.M. Ng, A.M. Sergent, S.N.G. Chu, Appl. Phys, Lett. 81 (2002) 3579.

[16] B.S. Simpkins, D.M. Schaadt, E.T. Yu, R.J. Molnar, J. Appl. Phys. 91 (2002) 9924

[17] B.D. Terris, J.E. Stern, D. Rugar, H.J. Mamin, Phys. Rev. Lett. 63 (1989) 2669

[18] C.I. Wu, A. Kahn, N. Taskar, D. Dorman, D. Gallagher, J. Appl. Phys. 83 (1998) 4249.

[19] S.M. Sze, Physics of Semiconductor Devices, Wiley, New York, 1981, pp. 270-275.

[20] H.C. Yang, T.Y. Lin, M.Y. Huang, Y.F. Chen, J. Appl. Phys. 86 (1999) 6124.

[21] N.M. Shmidt, V.V. Emtsev, A.S. Kryzhanovsky, R.N. Kyutt, W.V. Lundin, D.S. Poloskin, V.V. Ratnikov, A.V. Sakharov, A.N. Titkov, A.S. Usikov, P. Girard, Phys. Stat. Sol. (b) 216 (1999) 581 